\title{
A New Nonlinear Diffusion Equation Model for Noisy Image Segmentation
}

\author{
Bo Chen, ${ }^{1,2}$ Xiao-Hui Zhou, ${ }^{1}$ Li-Wei Zhang, ${ }^{3}$ Jie Wang, \\ Wei-Qiang Zhang, ${ }^{1}$ and Chen Zhang ${ }^{1}$ \\ ${ }^{1}$ College of Mathematics and Statistics, Shenzhen University, Shenzhen 518060, China
${ }^{2}$ Shenzhen Key Laboratory of Media Security, Shenzhen University, Shenzhen 518060, China
${ }^{3}$ School of Mechanical Engineering and Automation, Fuzhou University, Fuzhou 350116, China
${ }^{4}$ College of Computer Science and Technology, Beijing University of Technology, Beijing 100124, China
}

Correspondence should be addressed to Wei-Qiang Zhang; wqzhang@szu.edu.cn

Received 3 September 2015; Revised 21 December 2015; Accepted 3 January 2016

Academic Editor: Ricardo Weder

Copyright (c) 2016 Bo Chen et al. This is an open access article distributed under the Creative Commons Attribution License, which permits unrestricted use, distribution, and reproduction in any medium, provided the original work is properly cited.

\begin{abstract}
Image segmentation and image denoising are two important and fundamental topics in the field of image processing. Geometric active contour model based on level set method can deal with the problem of image segmentation, but it does not consider the problem of image denoising. In this paper, a new diffusion equation model for noisy image segmentation is proposed by incorporating some classical diffusion equation denoising models into the segmental process. An assumption about the connection between the image intensity and level set function is given firstly. Some classical denoising models are employed to describe the evolution of level set function secondly. The final nonlinear diffusion equation model for noisy image segmentation is built thirdly. Then image segmentation and image denoising are combined in a united framework. The segmental results can be presented by level set function. Experimental results show that the new model has the advantage of noise resistance and is superior to traditional segmentation model.
\end{abstract}

\section{Introduction}

Image segmentation and image denoising are basic topics in the fields of image processing. The results of image segmentation and denoising can influence the following image processing directly. They also play an important role in the fields of computer vision, target tracking, and so forth.

The target of image denoising is to recover the true image from the noisy one. Because noise and boundary of image both share high frequency, it is difficult to reduce the noise while containing the boundary of image. For the sake of solving this difficulty, a series of nonlinear denoising models [1-5] are proposed and total variation (TV) model [1] is an excellent one among them. TV model is a famous denoising model proposed by Rudin et al. and it has the ability to preserve image boundary. At the same time, the target of image segmentation is to find a curve which coincides with the true boundary of image object. Thousands of segmentation methods were proposed before. Active contour model (ACM) is the focus recently. ACM can be categorized as two classes. One is parameterized model $[6,7]$ and another is geometrical model [8-12]. Geometric active contour model also has two classes, gradient-based model and region-based model. Chan-Vese (CV) model [10] is a famous region-based geometric active contour model. It is more flexible for initial curve and can deal with images which have weak boundaries because it does not rely on the gradient information.

Image segmentation and image denoising are independent most of the time. In this paper, a new diffusion equation model for noisy image segmentation is proposed by incorporating some classical diffusion equation denoising models into the segmental process. An assumption about the connection between the image intensity and level set function is given firstly. Some classical denoising models are employed to describe the evolution of level set function secondly. The final nonlinear diffusion equation model for noisy image 
segmentation is built thirdly. Then image segmentation and image denoising are combined in a united framework. The segmental results can be presented by level set function. Experimental results show that the new model is available for noisy image segmentation.

This paper is organized as follows. The next section will introduce the related models. The new nonlinear diffusion equation model for noisy image segmentation is described in the third section. Experimental results are shown in the fourth section and we draw a conclusion in the fifth section.

\section{Related Models}

Four models will be introduced in this section. One is segmentation model and the other is denoising models.

2.1. CV Model. CV model is an important segmentation model and it is suitable for the image with intensity homogeneity. CV model assumes that the image is homogeneous and different objects in the image have different intensities. The corresponding energy function is as follows:

$$
\begin{aligned}
E\left(C, c_{1}, c_{2}\right)= & \mu_{0} \text { Length }(C) \\
& +\lambda_{1} \int_{\operatorname{inside}(C)}\left|u_{0}(x, y)-c_{1}\right|^{2} d x d y \\
& +\lambda_{2} \int_{\text {outside }(C)}\left|u_{0}(x, y)-c_{2}\right|^{2} d x d y,
\end{aligned}
$$

where $\mu_{0}$ is the nonnegative weight for length of curve $C, u_{0}$ is the image intensity, and $\lambda_{1}, \lambda_{2}$ are the weights of external energy term. $c_{1}$ and $c_{2}$ are the mean of gray values inside and outside the moving contour. In order to solve the topological changes of the curve $C$ easily, we introduce the level set function into (1):

$$
\begin{array}{ll}
\phi(x, y)<0, & (x, y) \in \text { outside }(C), \\
\phi(x, y)>0, & (x, y) \in \text { inside }(C), \\
\phi(x, y)=0, & (x, y) \in C .
\end{array}
$$

Then (1) also can be represented as follows:

$$
\begin{aligned}
E\left(\phi, c_{1}, c_{2}\right) & \\
= & \mu_{0} \int_{\Omega}|\nabla H(\phi)| d x d y \\
& +\lambda_{1} \int_{\Omega}\left|u_{0}(x, y)-c_{1}\right|^{2} H(\phi) d x d y \\
& +\lambda_{2} \int_{\Omega}\left|u_{0}(x, y)-c_{2}\right|^{2}(1-H(\phi)) d x d y,
\end{aligned}
$$

where $\Omega$ denotes the field of image. $H(\phi)$ is the Heaviside function. For making Heaviside function $H(\phi)$ differentiable, we replace $H(\phi)$ by $H_{\varepsilon}(\phi)$ as follows:

$$
\begin{aligned}
& H_{\varepsilon}(\phi)=\frac{1}{2}\left(1+\frac{2}{\pi} \arctan \left(\frac{\phi}{\varepsilon}\right)\right), \\
& \delta_{\varepsilon}(\phi)=H_{\varepsilon}^{\prime}(\phi)=\frac{1}{\pi} \frac{\varepsilon}{\varepsilon^{2}+\phi^{2}} .
\end{aligned}
$$

Then (3) can be written as follows:

$$
\begin{aligned}
E\left(\phi, c_{1}, c_{2}\right) & \\
= & \mu_{0} \int_{\Omega}\left|\nabla H_{\varepsilon}(\phi)\right| d x d y \\
& +\lambda_{1} \int_{\Omega}\left|u_{0}(x, y)-c_{1}\right|^{2} H_{\varepsilon}(\phi) d x d y \\
& +\lambda_{2} \int_{\Omega}\left|u_{0}(x, y)-c_{2}\right|^{2}\left(1-H_{\varepsilon}(\phi)\right) d x d y .
\end{aligned}
$$

By taking partial derivations for $c_{1}$ and $c_{2}$ in (5), we can get the expressions of $c_{1}$ and $c_{2}$ as follows:

$$
\begin{aligned}
& c_{1}=\frac{\int_{\Omega} u_{0}(x, y) H_{\varepsilon}(\phi) d x d y}{\int_{\Omega} H_{\varepsilon}(\varphi) d x d y}, \\
& c_{2}=\frac{\int_{\Omega} u_{0}(x, y)\left(1-H_{\varepsilon}(\phi)\right) d x d y}{\int_{\Omega}\left(1-H_{\varepsilon}(\phi)\right) d x d y} .
\end{aligned}
$$

The corresponding Euler-Lagrange equation of (5) is as follows:

$$
\begin{aligned}
& -\delta_{\varepsilon}(\phi) \\
& \cdot\left[\mu_{0} \operatorname{div}\left(\frac{\nabla \phi}{|\nabla \phi|}\right)-\lambda_{1}\left(u_{0}-c_{1}\right)^{2}+\lambda_{2}\left(u_{0}-c_{2}\right)^{2}\right] \\
& \quad=0 .
\end{aligned}
$$

The final level set evolution equation can be written as follows:

$$
\begin{aligned}
\frac{\partial \phi}{\partial t} & =\delta_{\varepsilon}(\phi) \\
& \cdot\left[\mu_{0} \operatorname{div}\left(\frac{\nabla \phi}{|\nabla \phi|}\right)-\lambda_{1}\left(u_{0}-c_{1}\right)^{2}+\lambda_{2}\left(u_{0}-c_{2}\right)^{2}\right] .
\end{aligned}
$$

2.2. TV Model. TV model is a classical model for image denoising and it utilizes $L_{1}$ norm of image gradient magnitude to replace $H_{1}$ norm of image gradient magnitude as the smooth term of image. TV model can protect the boundary of image while reducing the noise. The energy function of TV model is established as follows:

$$
E(u)=\int_{\Omega}|\nabla u| d x d y+\frac{\lambda}{2} \cdot \int_{\Omega}\left(u-u_{0}\right)^{2} d x d y,
$$

where the first term $\int_{\Omega}|\nabla u| d x d y$ is the smooth term of image and the second term $\int_{\Omega}\left(u-u_{0}\right)^{2} d x d y$ is the fidelity term of image. $u$ denotes the denoising image. $\Omega$ denotes the field of image. $|\nabla u|$ is the gradient magnitude of image. $\lambda \geq 0$ is the coefficient of fidelity term.

The Euler-Lagrange equation of (9) is as follows:

$$
-\operatorname{div}\left(\frac{\nabla u}{|\nabla u|}\right)+\lambda \cdot\left(u-u_{0}\right)=0 .
$$

Utilizing the gradient decent flow, we can get the partial difference equation as follows:

$$
\frac{\partial u}{\partial t}=\operatorname{div}\left(\frac{\nabla u}{|\nabla u|}\right)+\lambda \cdot\left(u_{0}-u\right) .
$$


2.3. Adaptive TV Model. Blomgren and Chan present a new norm which is different from the $L_{1}$ norm in [2]:

$$
R_{p}(u)=\int_{\Omega}|\nabla u|^{p(|\nabla u|)} d x d y,
$$

where the exponent $p$ depends on the module value $|\nabla u|$ of image gradient and satisfies these constrains as follows:

(1) decreasing monotonously,

(2) $p(x) \rightarrow 2$ when $x \rightarrow 0$ and $p(x) \rightarrow 1$ when $x \rightarrow \infty$.

Therefore, $R_{p}(u) \approx \int_{\Omega}|\nabla u|^{2} d x d y$ while lying in the flat region of image; namely, $|\nabla u|$ is very small. $|\nabla u|$ is very big when the pixel is near the boundary of object. At the same time, $p(|\nabla u|) \approx 1$ when $|\nabla u| \rightarrow \infty$. Therefore, $R_{p}(u) \approx$ $\int_{\Omega}|\nabla u| d x d y$ according to (12). That is to say, the new norm $R_{p}(u)$ would choose $L_{1}$ norm and $H_{1}$ norm automatically.

We can define $p(x)$ as follows:

$$
p(x)=\frac{2+x}{1+x} .
$$
follows:

The corresponding energy function can be expressed as

$$
E(u)=\int_{\Omega}|\nabla u|^{p(|\nabla u|)} d x d y+\frac{\lambda}{2} \cdot \int_{\Omega}\left(u-u_{0}\right)^{2} d x d y .
$$

Finally, the partial difference equation of image $u$ about time parameter $t$ can be written as follows:

$$
\frac{\partial u}{\partial t}=\operatorname{div}\left(q(|\nabla u|) \frac{\nabla u}{|\nabla u|}\right)+\lambda \cdot\left(u_{0}-u\right),
$$

where

$$
\begin{aligned}
q(|\nabla u|)= & p(|\nabla u|)|\nabla u|^{p(|\nabla u|)-1}+\ln |\nabla u| \cdot p^{\prime}(|\nabla u|) \\
& \cdot|\nabla u|^{p(|\nabla u|)} .
\end{aligned}
$$

Compared with (11), (15) has more computation complexities. For the sake of reducing the computation complexities, we replace $q(|\nabla u|)$ in (14) by the term $q\left(\left|\nabla\left(G_{\sigma} * u_{0}\right)\right|\right)$, where $G_{\sigma} * u_{0}$ denotes that the image $u_{0}$ is filtered by Gaussian filter of variation $\sigma$. Therefore, we do not need to update $q(|\nabla u|)$ every time when updating the denoising image $u$.

2.4. Anisotropic Diffusion Model. Catté et al. propose a denoising model based on anisotropic diffusion [13]. The final partial difference equation is as follows:

$$
\begin{aligned}
\frac{\partial u}{\partial t} & =\operatorname{div}\left(g\left(\left|\nabla\left(G_{\sigma} * u_{0}\right)\right|\right) \nabla u\right), \\
u(x, 0) & =u_{0},
\end{aligned}
$$

where

$$
g(s)=\frac{1}{1+s^{2} / K},
$$

where $K$ is a nonnegative number. The diffusion term can protect the boundary information and so the anisotropic diffusion model can obtain better results than the denoising model based on Laplace Operation.

\section{The New Nonlinear Diffusion Equation Model for Noisy Image Segmentation}

Inspired by CV model, we can know that the image with two objects can be described as follows:

$$
u=c_{1} \cdot H_{\varepsilon}(\phi)+c_{2} \cdot\left(1-H_{\varepsilon}(\phi)\right) .
$$

From (19), we can establish the connection between the image intensity and the level set function. Therefore, we can introduce the level set function into the energy function of denoising models. Averagely, we establish the energy function of denoising models as follows:

$$
E(u)=\operatorname{smooth}(u)+\lambda \cdot \operatorname{fidelity}(u),
$$

where $\operatorname{smooth}(u)$ is the smooth term, fidelity $(u)$ is the fidelity term, and $\lambda$ is the weight of the fidelity term.

Now we introduce (19) into (20) and the corresponding Euler-Lagrange equation can be written as follows:

$$
\frac{\partial E(u(\phi))}{\partial \phi}=\frac{\partial E}{\partial u} \cdot \frac{\partial u}{\partial \phi},
$$

where

$$
\frac{\partial u}{\partial \phi}=\left(c_{1}-c_{2}\right) \cdot \delta_{\varepsilon}(\phi)
$$

Finally, the corresponding level set equation is written as follows:

$$
\frac{\partial \phi}{\partial t}=-\frac{\partial E(u(\phi))}{\partial \phi}=-\frac{\partial E}{\partial u} \cdot \frac{\partial u}{\partial \phi}=\frac{\partial u}{\partial t} \cdot \frac{\partial u}{\partial \phi} .
$$

We will present three examples of segmentation model based on the related denoising models mentioned before.

3.1. TV-Based Segmentation Model. According to (23), we can formulate the level set equation based on TV model. The Euler-Lagrange equation of TV model is as follows:

$$
\frac{\partial E}{\partial u}=-\operatorname{div}\left(\frac{\nabla u}{|\nabla u|}\right)+\lambda \cdot\left(u-u_{0}\right)=0 .
$$

According to (11), (19), (23), and (24), we can obtain the final level set equation as follows:

$$
\begin{aligned}
\frac{\partial \phi}{\partial t} & =\delta_{\varepsilon}(\phi) \cdot\left(c_{1}-c_{2}\right)\left[\operatorname{div}\left(\frac{\left(c_{1}-c_{2}\right) \cdot \delta_{\varepsilon}(\phi) \cdot \nabla \phi}{\left|c_{1}-c_{2}\right| \cdot \delta_{\varepsilon}(\phi) \cdot|\nabla \phi|}\right)\right. \\
& \left.+\lambda\left(u_{0}-c_{1} \cdot H_{\varepsilon}(\phi)+c_{2} \cdot\left(1-H_{\varepsilon}(\phi)\right)\right)\right]=\delta_{\varepsilon}(\phi) \\
& +\left(c_{1}-c_{2}\right) \cdot\left[\operatorname{div}\left(\frac{\left(c_{1}-c_{2}\right) \cdot \nabla \phi}{\left|c_{1}-c_{2}\right| \cdot|\nabla \phi|}\right)\right. \\
& \left.+\lambda\left(u_{0}-c_{1} \cdot H_{\varepsilon}(\phi)+c_{2} \cdot\left(1-H_{\varepsilon}(\phi)\right)\right)\right] .
\end{aligned}
$$


3.2. Adaptive TV-Based Segmentation Model. Similar to the TV-based segmentation model, we can find the corresponding partial difference equation of image $u$ firstly and it is described in (15). Then utilizing (15), (19), and (23), we can obtain the Euler-Lagrange equation of adaptive TV-based segmentation model as follows:

$$
\begin{aligned}
& \frac{\partial E(u(\phi))}{\partial \phi}=\frac{\partial E}{\partial u} \cdot \frac{\partial u}{\partial \phi} \\
& =\left(\operatorname{div}\left(q\left(\left|\nabla\left(G_{\sigma} * u_{0}\right)\right|\right) \frac{\nabla u}{|\nabla u|}\right)+\lambda \cdot\left(u_{0}-u\right)\right) \\
& \quad \cdot\left(c_{1}-c_{2}\right) \cdot \delta_{\varepsilon}(\phi)=0 .
\end{aligned}
$$

The partial different equation of level set function is written as follows:

$$
\begin{aligned}
\frac{\partial \phi}{\partial t} & =\delta_{\varepsilon}(\phi) \cdot\left(c_{1}-c_{2}\right) \\
\cdot & {\left[\operatorname{div}\left(q\left(\left|\nabla\left(G_{\sigma} * u_{0}\right)\right|\right) \cdot \frac{\left(c_{1}-c_{2}\right) \cdot \delta_{\varepsilon}(\phi) \cdot \nabla \phi}{\left|c_{1}-c_{2}\right| \cdot \delta_{\varepsilon}(\phi) \cdot|\nabla \phi|}\right)\right.} \\
& \left.+\lambda\left(u_{0}-c_{1} \cdot H_{\varepsilon}(\phi)+c_{2} \cdot\left(1-H_{\varepsilon}(\phi)\right)\right)\right] .
\end{aligned}
$$

3.3. Anisotropic Diffusion-Based Segmentation Model. As described in Section 2.4, we can acquire the corresponding partial difference equation of image $u$ described in (17). For preserving the important image information, we introduce the fidelity term to this model and the final partial difference equation of image $u$ can be written as follows:

$$
\frac{\partial u}{\partial t}=\operatorname{div}\left(g\left(\left|\nabla\left(G_{\sigma} * u_{0}\right)\right|\right) \nabla u\right)+\lambda \cdot\left(u-u_{0}\right) .
$$

According to (17), (19), (23), and (29), we can obtain the final level set function as follows:

$$
\begin{aligned}
\frac{\partial \phi}{\partial t} & =\delta_{\varepsilon}(\phi) \cdot\left(c_{1}-c_{2}\right) \cdot[\operatorname{div}(g(|\nabla \phi|) \cdot \nabla \phi) \\
& \left.+\lambda\left(u_{0}-c_{1} \cdot H_{\varepsilon}(\phi)+c_{2} \cdot\left(1-H_{\varepsilon}(\phi)\right)\right)\right] .
\end{aligned}
$$

3.4. Reinitialization of Level Set Function. A signed distance penalizing energy functional is presented by Li et al. [14] as follows:

$$
P(\phi)=\frac{1}{2} \cdot \int_{\Omega}(|\nabla \phi|-1)^{2} d x d y .
$$

The corresponding gradient descent flow of $P(\phi)$ is as follows:

$$
\frac{\partial \phi}{\partial t}=\operatorname{div}\left((|\nabla \phi|-1) \cdot \frac{\nabla \phi}{|\nabla \phi|}\right)=\Delta \phi-\operatorname{div}\left(\frac{\nabla \phi}{|\nabla \phi|}\right) .
$$

Literatures $[15,16]$ show that a function with Laplace evolution is equivalent to using a Gaussian filter. Then we filter the initial conditions of the level set function with Gaussian kernel. So we can remove the curvature term $\operatorname{div}(\nabla \phi /|\nabla \phi|)$.
We can conclude the procedures of the presented model as follows:

(1) Initialize the level set function.

(2) If necessary, compute $q\left(\left|\nabla\left(G_{\sigma} * u_{0}\right)\right|\right), g\left(\left|\nabla\left(G_{\sigma} * u_{0}\right)\right|\right)$ by (16) and (18).

(3) Calculate $c_{1}, c_{2}$ by (6).

(4) Obtain the level set function $\phi$ by (25), (27), or (29).

(5) Smooth the level set function $\phi$ with Gaussian filter. If the level set function $\phi$ is not satisfied, return to procedure (3).

\section{Experimental Results}

In this section, we compare the segmental results of four models, CV model, TV-based segmentation model, adaptive TV-based segmentation model, and anisotropic diffusionbased segmentation model. In CV model, we set time step as 1 , space step as 1, iteration number as 1500, the length parameter as $\mu_{0}$, and the radius of initial circle as different value in different case. In the other models, we set time step and space step the same as CV model, $\sigma=0.5, K=500$, and iteration number as 1500 . Besides, we choose $\varepsilon$ as 1.5 for all models. Their initial curve is set as blue curves. The parameters of external energy $\lambda_{1}, \lambda_{2}$ are set as 1 for all models. The parameter $\lambda$ depends on the degree of noise.

Figure 1 shows the segmental results of a noisy image contaminated by Gaussian noise with variation 0.05. (a, e), $(b, f),(c, g)$, and $(d, h)$ represent the segmentation results of CV model, TV-based segmentation model, adaptive TVbased model, and anisotropic diffusion-based segmentation model, respectively. The initial curve of all models is a circle as Figure 1(a) shows and its radius is equal to 20. In TVbased segmentation model, adaptive TV-based model, and anisotropic diffusion-based segmentation model, we choose $\lambda=0.01$. Obviously, the new segmentation model incorporating with denoising models is better than the original $\mathrm{CV}$ model.

Figure 2 shows the segmental results of a noisy image contaminated by Gaussian noise with variation as 0.1 . $(\mathrm{a}, \mathrm{e})$, $(b, f),(c, g)$, and $(\mathrm{d}, \mathrm{h})$ represent the segmentation result of CV model, TV-based segmentation model, adaptive TVbased model, and anisotropic diffusion-based segmentation model, respectively. The initial curve of all models is a circle as in Figure 2(a) and its radius is set as 20. In TV-based segmentation model, adaptive TV-based model, and anisotropic diffusion-based segmentation model, we choose $\lambda=0.01$. Obviously, the new segmentation model based on denoising algorithm can get satisfied contours for noisy image.

Figure 3 gives the segmental results of a galaxy image contaminated by Gaussian noise with variation of 0.05 . (a, e), $(b, f),(c, g)$, and $(d, h)$ represent the segmentation result of CV model, TV-based segmentation model, adaptive TVbased model, and anisotropic diffusion-based segmentation model, respectively. The initial curve of all models is a circle as in Figure 3(a) and its radius is set as 50. In TVbased segmentation model and adaptive TV-based model, we choose $\lambda=0.02$. In anisotropic diffusion-based segmentation 


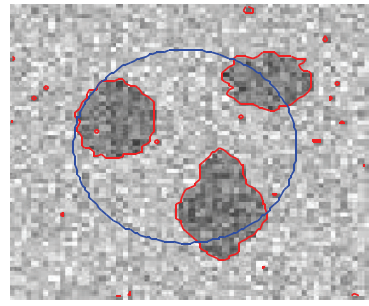

(a)

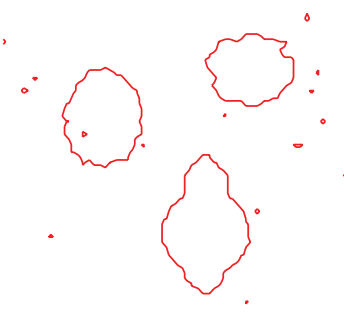

(e)

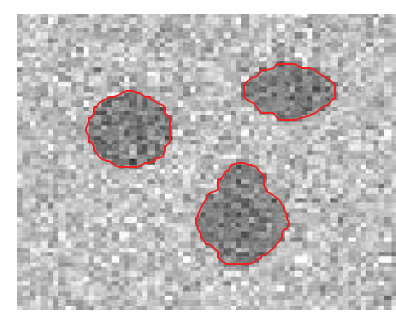

(b)
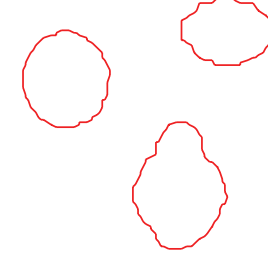

(f)



(c)

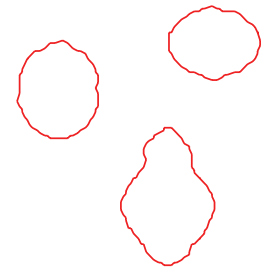

(g)

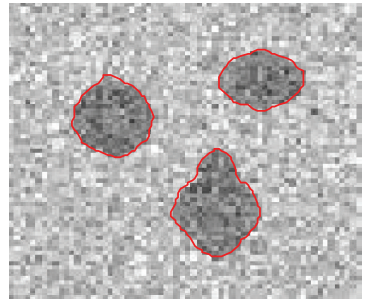

(d)

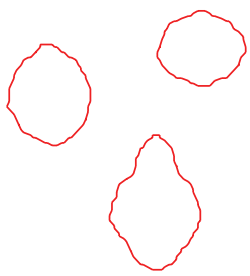

(h)

FIGURE 1: Segmental results of a noisy image with Gaussian variance 0.05. (a) Segmental result with CV model and the blue curve is the initial contour $\left(\mu_{0}=0.02 * 255 * 255\right)$. (b) Segmental result with TV-based segmentation model. (c) Segmental result with adaptive TV-based model. (d) Anisotropic diffusion-based segmentation model. (e), (f), (g), and (h) are the corresponding contours to (a), (b), (c), and (d), respectively.

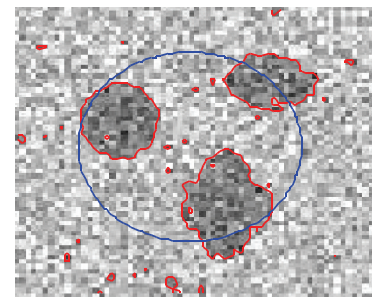

(a)

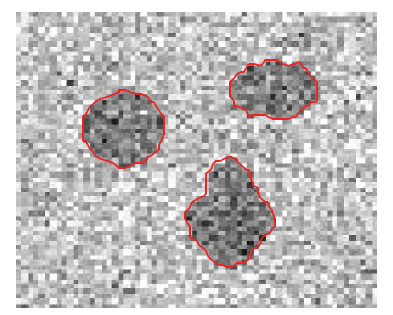

(b)

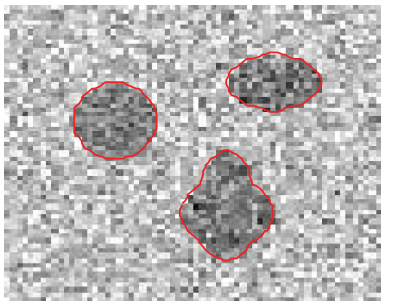

(c)

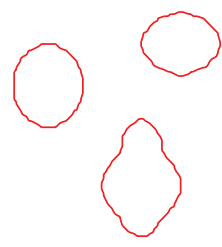

(g)

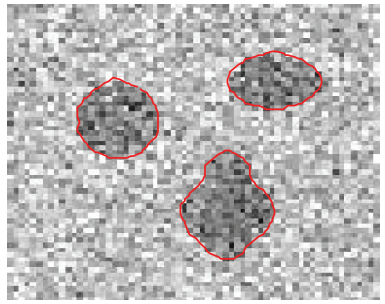

(d)



(h)

FIgURE 2: Segmental results of a noisy image with Gaussian variance 0.1. (a) Segmental result with CV model and the blue curve is the initial contour ( $\mu_{0}=0.03 * 255 * 255$ ). (b) Segmental result with TV-based segmentation model. (c) Segmental result with adaptive TV-based model. (d) Anisotropic diffusion-based segmentation model. (e), (f), (g), and (h) are the corresponding contours to (a), (b), (c), and (d), respectively.

model, we choose $\lambda=0.005$. As shown in Figure 3(a), the result of $\mathrm{CV}$ model obviously extracts the spots produced by Gaussian noise. Our models can obtain the satisfied contour without noisy spots.

Figure 4 shows the segmental results of a galaxy image contaminated by Gaussian noise with variation of 0.1 . $(\mathrm{a}, \mathrm{e})$, $(b, f),(c, g)$, and $(d, h)$ represent the segmentation results of $\mathrm{CV}$ model, TV-based segmentation model, adaptive
TV-based segmentation model, and anisotropic diffusionbased segmentation model, respectively. The initial curve of all models is a circle shown in Figure 4(a) and its radius is set as 70. In TV-based segmentation model and adaptive TV-based segmentation model, we choose $\lambda=0.02$. In anisotropic diffusion-based segmentation model, we choose $\lambda=0.005$. Obviously, our models can get satisfied contour of objects in galaxy image. 




(a)

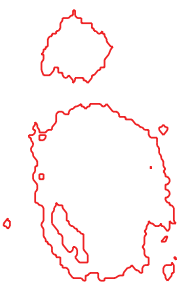

(e)

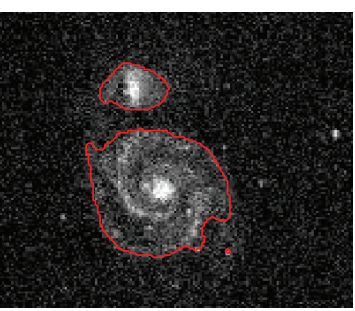

(b)

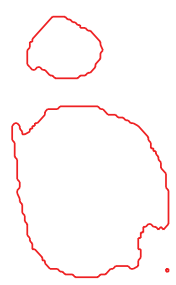

(f)

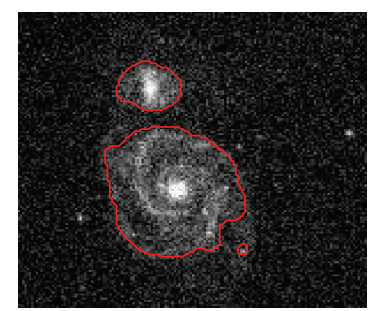

(c)

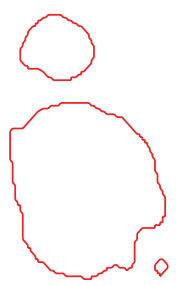

(g)



(d)



(h)

FIGURE 3: Segmental results of a galaxy image with Gaussian variance 0.05. (a) Segmental result with CV model and the blue curve is the initial contour $\left(\mu_{0}=0.03 * 255 * 255\right)$. (b) Segmental result with TV-based segmentation model. (c) Segmental result with adaptive TV-based model. (d) Anisotropic diffusion-based segmentation model. (e), (f), (g), and (h) are the corresponding contours to (a), (b), (c), and (d), respectively.



(a)

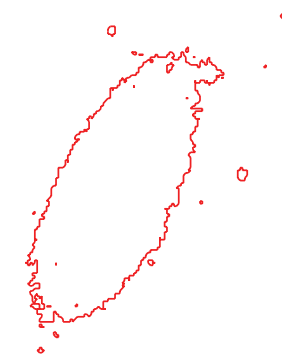

(e)

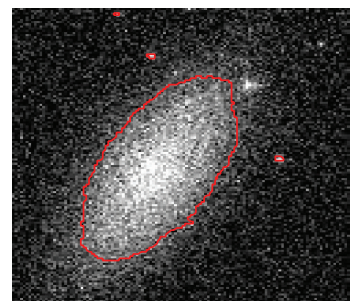

(b)

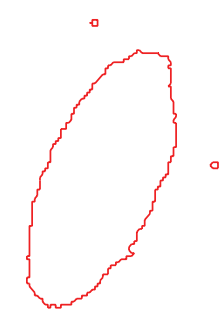

(f)

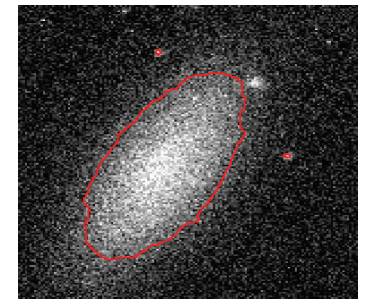

(c)

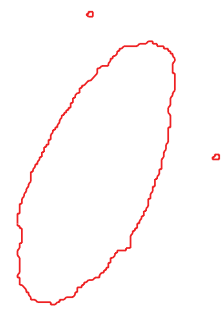

(g)

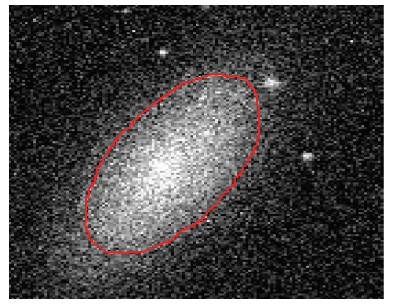

(d)

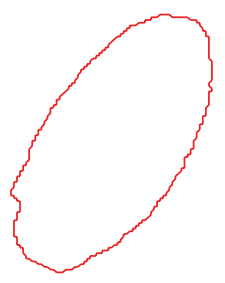

(h)

FIGURE 4: Segmental results of a galaxy image with Gaussian variance 0.1. (a) Segmental result with CV model and the blue curve is the initial contour $\left(\mu_{0}=0.04 * 255 * 255\right)$. (b) Segmental result with TV-based segmentation model. (c) Segmental result with adaptive TV-based model. (d) Anisotropic diffusion-based segmentation model. (e), (f), (g), and (h) are the corresponding contours to (a), (b), (c), and (d), respectively.

\section{Conclusion}

Image segmentation and image denoising are all important in the field of image processing, but they are independent most of the time. In this paper, a new diffusion equation model for noisy image segmentation is proposed by incorporating some classical diffusion equation denoising models into the segmental process. An assumption about the connection between the image intensity and level set function is given. Some classical denoising models are employed. Image 
segmentation and image denoising are combined in a united framework. The segmental results can be presented by level set function. Experimental results show that the new segmentation model has the advantage of noise resistance and is superior to traditional segmentation model.

\section{Competing Interests}

The authors declare that they have no competing interests.

\section{Acknowledgments}

This work is partially supported by Guangdong Provincial Key Laboratory of Medical Image Processing (2014B030301042), Science and Technology Planning Project of Shenzhen City (JCYJ20140828163633997), National Natural Science Foundation of China (61272252, 81371544, and 61472257), the HD Video R\&D Platform for Intelligent Analysis and Processing in Guangdong Engineering Technology Research Centre of Colleges and Universities (GCZX-A1409), the National Science Technology Major Project of the Ministry of Science and Technology of China (2014BAI17B02), and Guangdong Provincial Science and Technology Plan Project (2013B040403005).

\section{References}

[1] L. I. Rudin, S. Osher, and E. Fatemi, "Nonlinear total variation based noise removal algorithms," Physica D: Nonlinear Phenomena, vol. 60, no. 1-4, pp. 259-268, 1992.

[2] P. Blomgren and T. F. Chan, "Color TV: total variation methods for restoration of vector-valued images," IEEE Transactions on Image Processing, vol. 7, no. 3, pp. 304-309, 1998.

[3] S. Esedoglu and S. J. Osher, "Decomposition of images by the anisotropic Rudin-Osher-Fatemi model," Communications on Pure and Applied Mathematics, vol. 57, no. 12, pp. 1609-1626, 2004.

[4] T. F. Chan, S. Osher, and J. Shen, "The digital TV filter and nonlinear denoising," IEEE Transactions on Image Processing, vol. 10, no. 2, pp. 231-241, 2001.

[5] B. Chen, J.-L. Cai, W. Chen, and Y. Li, "A multiplicative noise removal approach based on partial differential equation model," Mathematical Problems in Engineering, vol. 2012, Article ID 242043, 14 pages, 2012.

[6] C. Xu and J. L. Prince, "Snakes, shapes, and gradient vector flow," IEEE Transactions on Image Processing, vol. 7, no. 3, pp. 359-369, 1998.

[7] L. D. Cohen and I. Cohen, "Finite-element methods for active contour models and balloons for 2-D and 3-D images," IEEE Transactions on Pattern Analysis and Machine Intelligence, vol. 15, no. 11, pp. 1131-1147, 1993.

[8] V. Caselles, F. Catté, T. Coll, and F. Dibos, "A geometric model for active contours in image processing," Numerische Mathematik, vol. 66, no. 1, pp. 1-31, 1993.

[9] V. Caselles, R. Kimmel, and G. Sapiro, "Geodesic active contours," International Journal of Computer Vision, vol. 22, no. 1, pp. 61-79, 1997.

[10] T. F. Chan and L. A. Vese, "Active contours without edges," IEEE Transactions on Image Processing, vol. 10, no. 2, pp. 266-277, 2001.
[11] K. Zhang, L. Zhang, H. Song, and W. Zhou, "Active contours with selective local or global segmentation: a new formulation and level set method," Image and Vision Computing, vol. 28, no. 4, pp. 668-676, 2010.

[12] B. Chen, Q.-H. Zou, W.-S. Chen, and Y. Li, "A fast region-based segmentation model with Gaussian kernel of fractional order," Advances in Mathematical Physics, vol. 2013, Article ID 501628, 7 pages, 2013.

[13] F. Catté, P.-L. Lions, J.-M. Morel, and T. Coll, "Image selective smoothing and edge detection by nonlinear diffusion," SIAM Journal on Numerical Analysis, vol. 29, no. 1, pp. 182-193, 1992.

[14] C. Li, C. Xu, C. Gui, and M. D. Fox, "Level set evolution without re-initialization: a new variational formulation," in Proceedings of the IEEE Computer Society Conference on Computer Vision and Pattern Recognition (CVPR '05), vol. 1, pp. 430-436, IEEE, San Diego, Calif, USA, June 2005.

[15] Y. Shi and W. Karl, "Real-time tracking using level sets," in Proceedings of the IEEE Conference on Computer Vision and Pattern Recognition (CVPR '05), vol. 2, pp. 34-41, June 2005.

[16] P. Perona and J. Malik, "Scale-space and edge detection using anisotropic diffusion," IEEE Transactions on Pattern Analysis and Machine Intelligence, vol. 12, no. 7, pp. 629-639, 1990. 


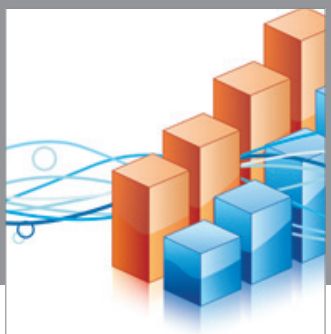

Advances in

Operations Research

vatem alat4

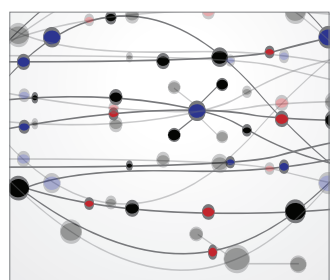

\section{The Scientific} World Journal
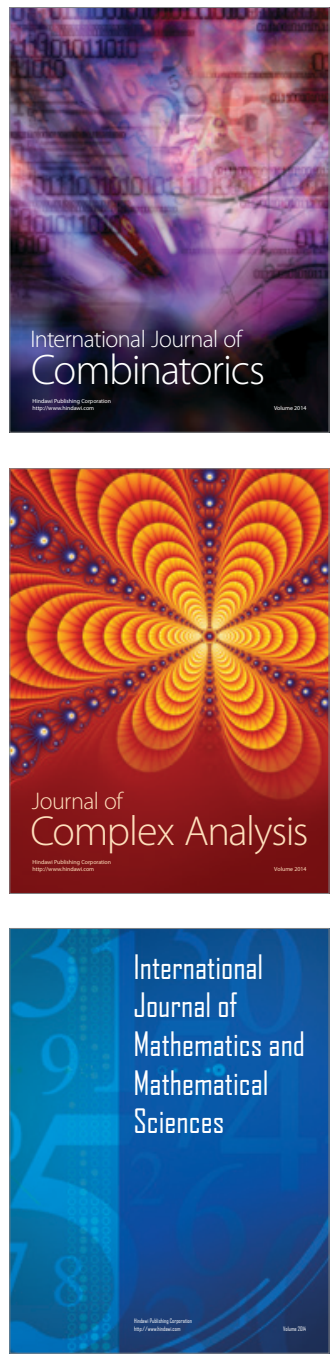
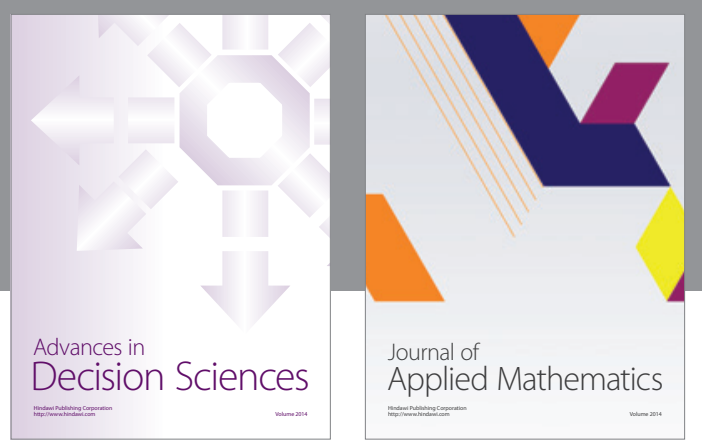

Algebra

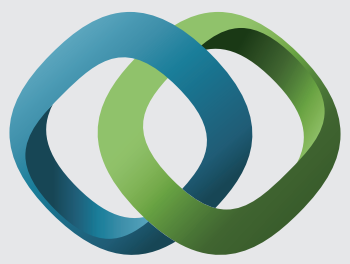

\section{Hindawi}

Submit your manuscripts at

http://www.hindawi.com
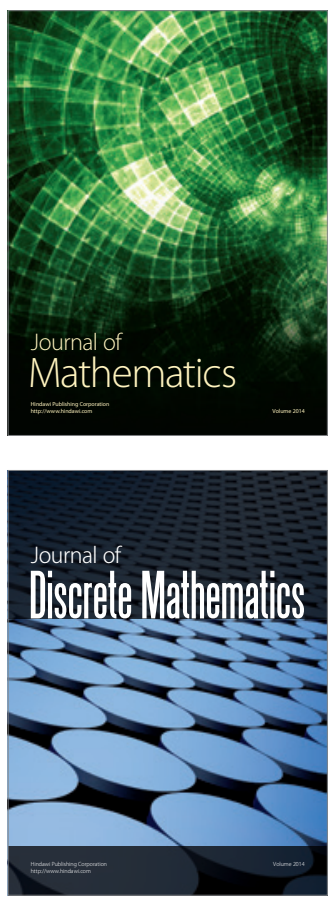

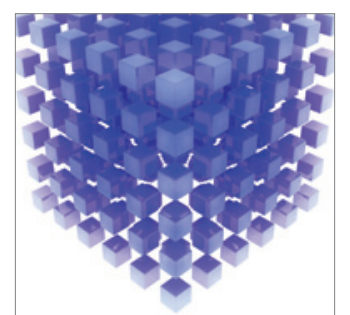

Mathematical Problems in Engineering
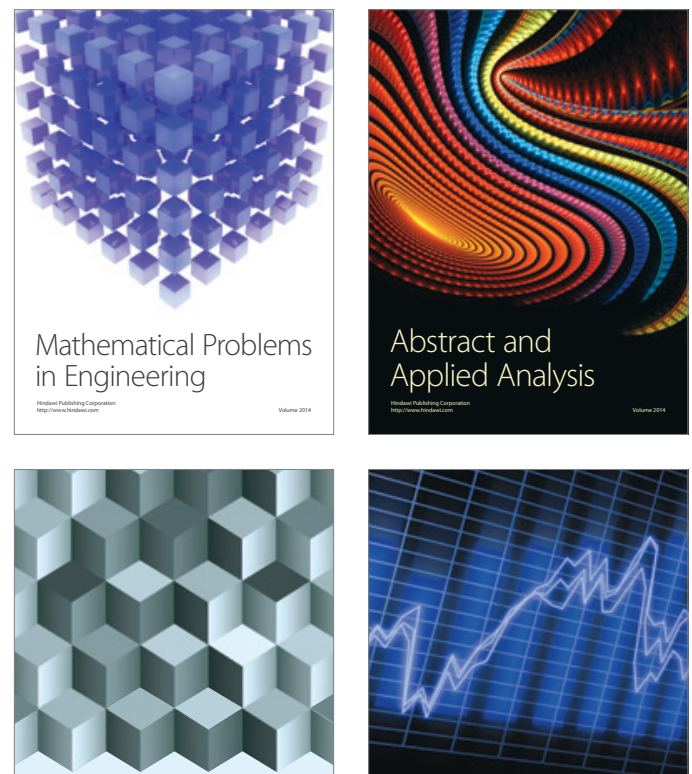

Journal of

Function Spaces

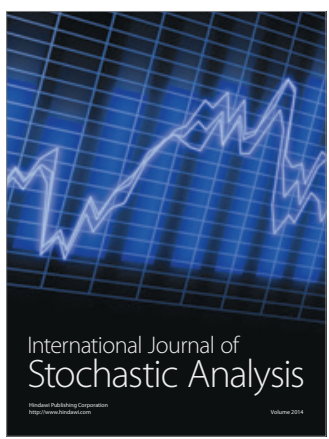

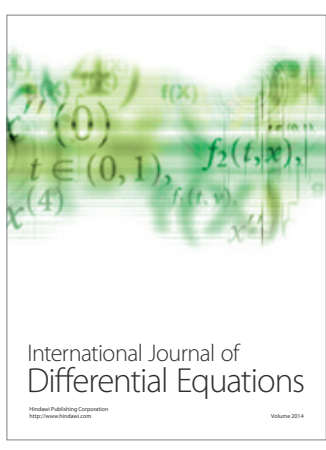
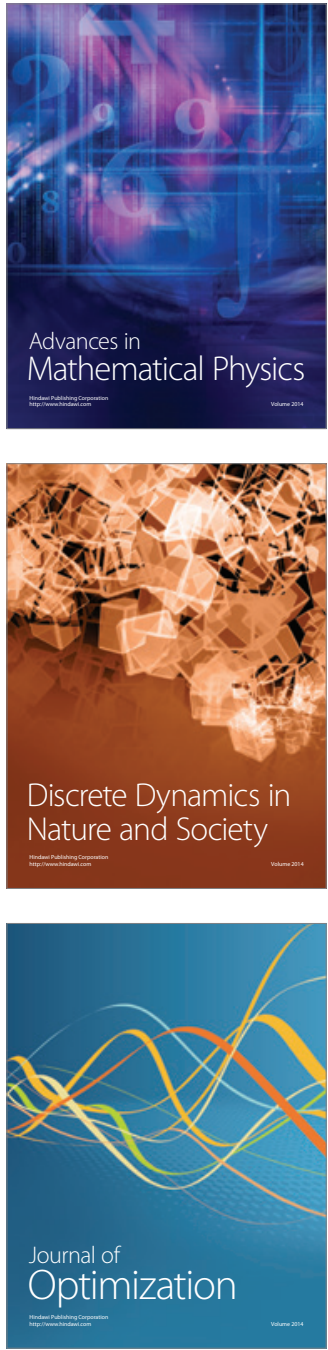